Chirurg 2016 $\cdot 87: 269-270$

DOI 10.1007/s00104-016-0152-y

Online publiziert: 9. Februar 2016

(c) Springer-Verlag Berlin Heidelberg 2016
Henning Dralle

Universitätsklinik für Allgemein-, Viszeral- und Gefäßchirurgie, Medizinische

Fakultät, Universitätsklinikum Halle, Halle (Saale), Deutschland

\title{
Gastroduodenale und rektale neuroendokrine Neoplasien
}

\author{
Wann endoskopische, wann \\ chirurgische Therapie?
}

Die in den letzten Jahren erhöhte „awareness" für neuroendokrine Neoplasien und die Zunahme der endoskopischen Diagnostik am oberen und unteren Gastrointestinaltrakt haben zu einer teils erheblichen Zunahme der Diagnose vor allem der kleinen neuroendokrinen Neoplasien (NEN) in diesem Bereich geführt. Unabhängig von der Frage, ob es sich nicht nur um eine $\mathrm{Zu}$ nahme der Inzidenz, sondern auch der Prävalenz handelt, ist es vor allem die gegenüber den ortsständigen Karzinomen differente Tumorbiologie, die im Einzelfall bei Vorliegen einer NEN die Therapieentscheidung schwierig machen kann und am besten interdisziplinär diskutiert wird.

\) Magen- und Duodenum-NEN $<1-2 \mathrm{~cm}$ können meist
endoskopisch entfernt werden

Das vorliegende Schwerpunktthema wurde ausgewählt, weil im Bereich der zunehmend häufiger diagnostizierten frühen, 1-2 cm großen NEN sowohl endoskopische als auch operative Behandlungsmöglichkeiten bestehen, jedoch dazu nur wenige und meist retrospektive Vergleichsstudien vorliegen. Eine Standortbestimmung der Entscheidungskriterien für bzw. gegen ein primär operatives Vorgehen ist daher von aktuell praktischer Relevanz zumal eine interdisziplinäre Diskussion dieses wichtigen Themas in der Zeitschrift Der Chirurg bislang gefehlt hat.
Im gastroenterologischen Beitrag von Maasberg et al. zu den gastroduodenalen NEN werden die 4 verschiedenen Typen der NEN des Magens und die 5 Typen der duodenalen NEN vorgestellt. Das therapeutische Fazit für die gastroduodenalen NEN wird klar formuliert: Die am häufigsten vorkommenden Typ-1-NEN des Magens sind wie diejenigen des Typ 2 bei einer Größe bis zu $2 \mathrm{~cm}$ eine Domäne der endoskopischen Therapie (endoskopische Mukosa- [EMR] bzw. Submukosa- [ESD] Resektion), für die Typen 3 und 4 sowie Typ-1- und Typ-2-Tumoren über $2 \mathrm{~cm}$ wird ein primär operatives Vorgehen empfohlen. Bei den Typ-1- und Typ-2-Tumoren wird von chirurgischer Seite (Fendrich und Bartsch) darüber hinaus bei submukosaüberschreitendem Wachstum, Angioinvasion oder Lymphknotenmetastasenverdacht die Indikation zum primär operativen Vorgehen gesehen. Bezüglich der insgesamt seltener reportierten duodenalen NEN besteht Einigkeit im gastroenterologisch-chirurgischen Konzept, dass bei Fehlen von Risikofaktoren (G2; Angioinvasion; Muscularispropria-Infiltration; Größe > $2 \mathrm{~cm}$ ) extraperiampulläre NEN endoskopisch abgetragen werden sollten, ansonsten und auch bei periampullärer Lokalisation der primär operative Weg zu bevorzugen ist. Bei günstiger Lage und entsprechender Erfahrung kann bei operativem Vorgehen auch laparoskopisch reseziert werden. Im Einzelfall wird vor allem bei periampullärer Lage die jeweilige Erfahrung des Gastroenterologen bzw. Chirurgen für die Therapiewahl ausschlaggebend sein.
Ebenso wie bei den gastroduodenalen NEN nimmt das Lymphknotenmetastasenrisiko auch bei den rektalen NEN mit zunehmender Tumorgröße, abnehmendem Differenzierungsgrad, Anstieg der Wachstumsfraktion, lymphovaskulärer Invasion und Infiltration in die Muscularis propria signifikant zu (Eick et al.; RadulovaMauersberger et al.). Während für die NEN des Rektums $<1 \mathrm{~cm}$ bei Fehlen der genannten Risikofaktoren einheitlich von gastroenterologischer und chirurgischer Seite endoskopische Abtragungs- bzw. Resektionsverfahren als adäquat angesehen werden, sprechen die aggregierten Daten neuerer asiatischer und US-amerikanischer Studien (s. Beitrag Radulova-Mauersberger et al.) für ein deutlich ansteigendes Lymphknotenmetastasenrisiko bereits ab Tumorgrößen von über $1,5 \mathrm{~cm}(<1 \mathrm{~cm}: 2-8 \% ; 1-2 \mathrm{~cm}$ : 24-70\%; > 2 cm: 50-80\%). Hieraus könnte der Schluss gezogen werden, dass rektale NEN in der Größenordnung von 1-2 cm ein höheres Metastasierungsrisiko besitzen als in der Regel gastroduodenale NEN (abgesehen von den Typ3/4-Magen-NEN) und daher bei diesen Tumoren eher ein radikal-operatives Vorgehen zu favorisieren wäre. Beide Autorengruppen plädieren daher für ein individuelles, interdisziplinäres und mit dem Patienten abgestimmtes Vorgehen.

Als Fazit der insgesamt sehr sorgfältig ausgelegten Beiträge kann geschlussfolgert werden, dass trotz der begrenzten Evidenzlage bei gastroduodenalen und rektalen NEN $<1 \mathrm{~cm}$ mit 
Fehlen von Risikofaktoren das endoskopische Vorgehen Therapie der Wahl ist, bei größeren Tumoren, Risikofaktoren oder inkompletter Abtragung dagegen operative Resektionsverfahren indiziert sind. Einer weiteren Klärung - und damit bevorzugtes Ziel interdisziplinärer Abstimmung - bedürfen die rektalen NEN $>1 \mathrm{~cm}$, die offenbar ein höheres Metastasierungsrisiko besitzen als gastroduodenale NET gleicher Größe.

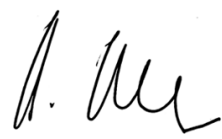

Prof. Dr. med. Dr. h. c. mult. Henning Dralle

\section{Korrespondenzadresse}

Prof. Dr. Dr. h. c. mult. H. Dralle FRCS, FACS, FEBS

Universitätsklinik für Allgemein-, Viszeral- und Gefäßchirurgie, Medizinische Fakultät, Universitätsklinikum Halle Ernst-Grube-Straße 40, 06097 Halle (Saale) henning.dralle@uk-halle.de

Interessenkonflikt. H. Dralle gibt an, dass kein Interessenkonflikt besteht.

\title{
Julius-Springer-Preis für Gefäßmedizin 2016
}

\author{
Der Brückenschlag von Forschungsarbeit zum \\ klinischen Nutzen wird ausgezeichnet
}

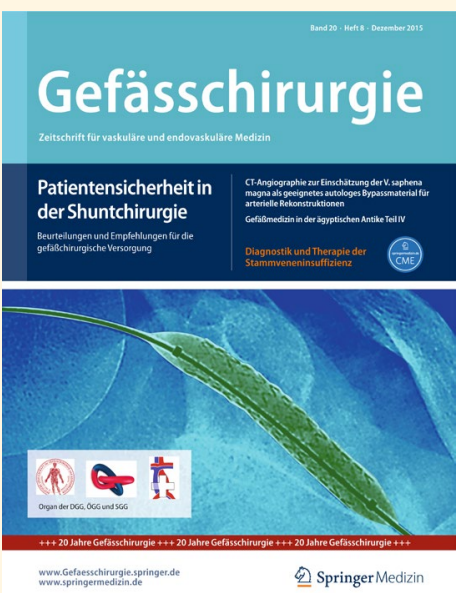

Die Gefäßmedizin ist ein Wachstumsfach mit zunehmender Bedeutung im Gesamtkonzept der Diagnostik und Therapie von Gefäßerkrankungen. Die wissenschaftliche Leitung von Gefässchirurgie mit Mitgliedern der deutschen, österreichischen und schweizerischen Gesellschaften für Gefäßchirurgie möchten zusammen mit dem SpringerVerlag die wissenschaftliche Forschung auf diesem Gebiet unterstützen und insbesondere den Nachwuchs durch die Verleihung des 7. Julius-Springer-Preises für Gefäßmedizin fördern.

Nutzen Sie die Gelegenheit und reichen Sie ein Exposé bis zum 15. September 2016 ein.

\section{Mit dem Preisgeld die Gewinnerarbeit Open Access stellen}

Mit dem Preisgeld im Wert einer Open Choice-Publikation und einer Übersetzung kann der Gewinner seinen Beitrag Open Access stellen. Der Artikel ist damit frei zugänglich und in der Datenbank PubMed Central im Volltext abrufbar.

\section{Teilnahmebedingungen}

- Der Autor soll ein Exposé auf Grundlage einer eigenen klinischen oder experimentellen Arbeit verfassen. Die Jury legt besonderen Wert auf den edukativen Charakter des Artikels sowie die Herausarbeitung der klinischen Relevanz der Beitrag soll eine Brücke von den erhobenen Daten zur klinischen Anwendung schlagen.

- Der Sieger wird gebeten, einen Übersichtsbeitrag in deutscher oder englischer Sprache zu verfassen, der in Gefässchirurgie zweisprachig und Open Choice publiziert wird.

- Alle anderen Autoren sind frei, ihren Beitrag anderweitig zu publizieren.

Der Siegerbeitrag wird durch eine Jury aus dem Herausgebergremium unter Regie der Schriftleitung bestimmt.

\section{Preisverleihung im Rahmen der Dreiländertagung}

Die Verleihung des 7. Julius-Springer-Preises für Gefäßmedizin erfolgt im Rahmen der Dreiländertagung der Schweizerischen, Deutschen und Österreichischen Gesellschaften für Gefäßchirurgie vom 5.-8. Oktober 2016 in Bern.

Bitte reichen Sie Ihr Exposé in elektronischer Form bei der Verlagsredaktion bis zum 15. September ein: tina.suhai@springer.com 